UCRL-JC- 120828

PREPRINT

\title{
Multiple Mechanisms In the Thermally Activated Plastic Flow of Tantalum
}

\author{
W. H. Gourdin \\ D. H. Lassila
}

This paper was prepared for submittal to 1995 APS Topical Conference, Seattle, WA August 13-18, 1995

June 27, 1995

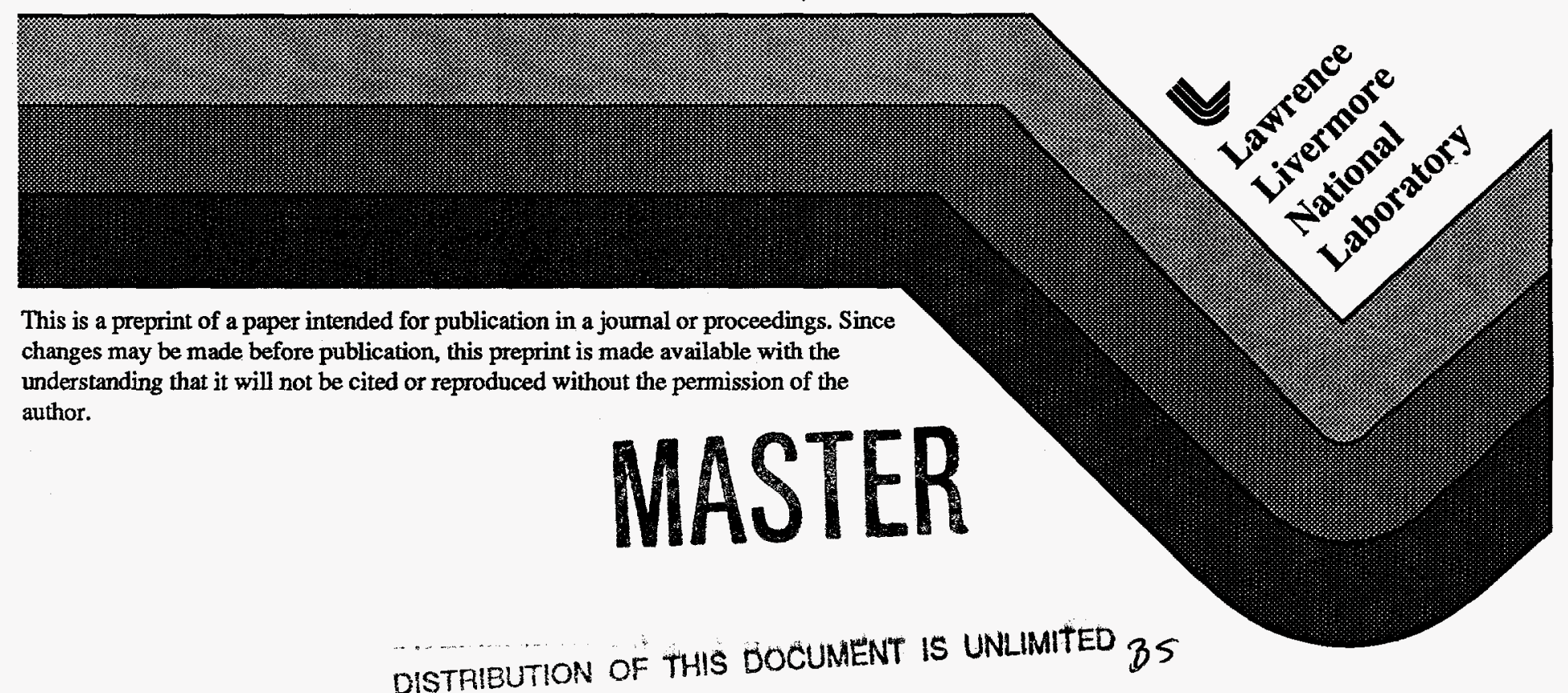




\section{DISCLAIMER}

Portions of this document may be illegible in electronic image products. Images are produced from the best available original document. 


\title{
MULTIPLE MECHANISMS IN THE THERMALLY ACTIVATED PLASTIC FLOW OF TANTALUM
}

\author{
William H. Gourdin and David H. Lassila \\ Chemistry \& Materials Science Department, University of California, \\ Lawrence Livermore National Laboratory, Livermore, California 94551
}

\begin{abstract}
We argue that many of the features of the plastic flow behavior of tantalum can be described by a model that incorporates a two-component Peierls-type mechanism and an fcc-like obstacle mechanism in series. We compare the results of calculations based on such a model with flow data for unalloyed tantalum over a wide range of strain rates and a modest range of temperatures.
\end{abstract}

\section{INTRODUCTION}

As in other body-centered-cubic (bcc) metals, the mechanical properties of tantalum display marked temperature and strain-rate dependences (1-3). Based on observations of tungsten alloys, we recently suggested (4) that the presence of tungsten somehow suppresses the "Peierls" mechanism that dominates the initial flow stress, leaving only an "obstacle" mechanism similar to that found in facecentered-cubic (fcc) metals (5-7). From this, we infer that unalloyed tantalum plastic flow occurs via a series combination of thermally activated "Peierls" and "obstacle" barriers that are comparable in magnitude and hence dominate the flow in different regimes of deformation, strain rate, and temperature. Because the mechanical threshold associated with the obstacle mechanism increases with deformation, work hardening appears in a natural way. In this paper, we explore the viability of such a model by comparing calculated results to flow curves for unalloyed tantalum over a wide strain-rate range and a modest temperature range.

\section{MODEL DESCRIPTION}

To propagate plastic flow, a dislocation must overcome both obstacles and Peieris barriers, as illustrated in Fig. 1. The total transit time between two obstacles is the sum of the time needed to overcome the first obstacle and all the Peieris barriers in between. Assuming Boltzmann probabilities for overcoming the energy barriers. the transit time is

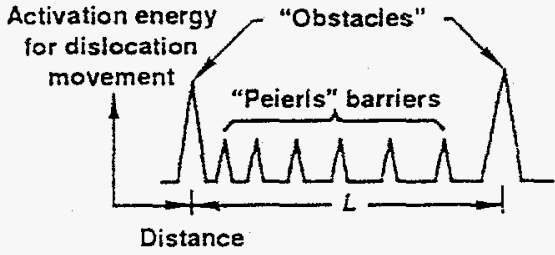

FIGURE 1. Schematic of two bariers to dislocation motion in series.

$$
t_{\mathrm{T}}=\left(\frac{1}{v_{0}}\right) \exp \left(\frac{G_{\mathrm{o}}}{k T}\right)+\left(\frac{1}{v_{\mathrm{p}}}\right) n_{\mathrm{p}} \exp \left(\frac{G_{\mathrm{p}}}{k T}\right)
$$

where $\nu_{0}$ and $\nu_{\mathrm{p}}$ are the attempt frequencies and $n_{\mathrm{p}}$ is the number of Peieris barriers. The strain rate is proportional to the average dislocation speed $L / h \mathrm{~T}$ :

$$
\dot{\varepsilon}=\frac{\dot{\varepsilon}_{0}}{\exp \left(\frac{G_{0}}{k T}\right)+P \exp \left(\frac{G_{p}}{k T}\right)}
$$

Equation (2) is the fundamental expression of the model that we propose for unalloyed tantalum. *

The activation free energy of the initial flow stress of tantalum as a function of the thermal stress $\sigma_{t}=\sigma-\sigma_{2}\left(\sigma_{a}\right.$ is the athermal stress) is shown in Fig. 2 (1). To reproduce the apparent rapid increase in $G_{\mathrm{p}}$ for very small $\sigma_{\mathrm{l}}$, we used the expression

\footnotetext{
* Dislocation drag could casily be included in this expression. but it has litule influence under the conditions of our experiments.
} 


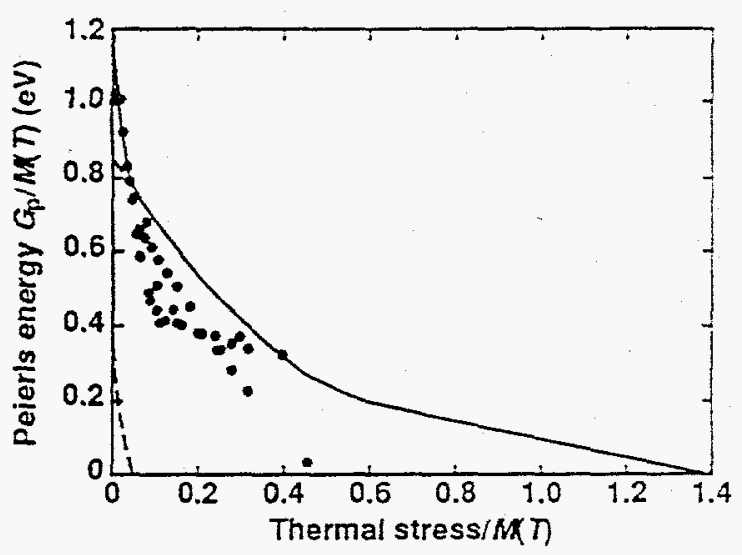

FIGURE 2. Activation energy at yield for unailoyed tantalum as a function of the thermal portion of the applied stress.

$$
\begin{aligned}
G_{p}= & G_{p 1} f\left(\frac{\sigma_{t}}{M(T) \hat{\sigma}_{p 1}}\right) \\
& +G_{p 2}\left[1-\left(\frac{\sigma_{t}}{M(T) \hat{\sigma}_{p 2}}\right)\right]^{2},
\end{aligned}
$$

where the secondary energy $G_{\mathrm{p} 2}$ and stress $\hat{\sigma}_{\mathrm{p} 2}$ are selected to agree with the data in Fig. 2.* We associate the first term with the Peierls energy reported in previous work, and the threshold stress associated with it, $\hat{\sigma}_{\mathrm{pl}}$, does not change with deformation. The function $f$ in this first term is close to the parabolic forms used elsewhere (2), but it deviates

\footnotetext{
* In this expression, the factor $M(T)=\mu(t) / \mu\left(T_{295}\right)$ nomalizes the stresses to the temperature-dependent shear modulus.
}

from this at large stresses to accommodale observed behavior at low temperatures and high strain rates.

The "obstacle" term in Eq. (2) is treated in a manner identical with that for copper $(6,7)$. The energy of activation over the barrier is given by

$$
G_{0}=G_{00}\left[1-\left(\frac{\sigma_{l}}{M(T) \hat{\sigma}_{0}}\right)^{p}\right]^{q} .
$$

The mechanical threshold $\sigma_{0}$ increases with deformation as prescribed by the empirical expression $(6,7)$

$$
\frac{d \hat{\sigma}_{o}}{d \varepsilon}=\theta_{0}\left[1-\frac{\tanh \left(\frac{2 \hat{\sigma}_{o}}{\hat{\sigma}_{o s}}\right)}{\tanh (2)}\right]
$$

and $\hat{\sigma}_{\text {os }}$ is given by

$$
\hat{\sigma}_{\text {os }}=\hat{\sigma}_{\text {os } 0}\left(\frac{\dot{\varepsilon}}{\dot{\varepsilon}_{s 0}}\right)^{1 / n} \text {. }
$$

where $n=\mu(T) b^{3} \mathrm{~A} / k T$.

Equations (2) and (4)-(6) as well as a specified form for the function $f$ in $\mathrm{Eq}$. (3) constitute the mathematical realization of the simple concept of

\begin{tabular}{|c|c|c|}
\hline Quantity & Description & Value/units \\
\hline$\mu$ & Temperature-dependent shear modulus: $\mu=\mu_{0}\left[1+W_{\mu}(T-300)\right]$ & GPa \\
\hline$\mu_{0}$ & Shear modulus at $300 \mathrm{~K}$ & 69 \\
\hline$W_{\mu}$ & $d u d d T$ at $300 \mathrm{~K}$ & $-2.94 \times 10^{-1} \mathrm{~K}^{-\mathrm{i}}$ \\
\hline$\dot{c}_{p}$ & Temperature-dependent specific heat: $c_{p}=(4.1868 / M)\left(B+10^{-3} \mathrm{Cn}\right\rangle$ & $J / k g / K$ \\
\hline$M$ & Molecular weight & $180.948 \times 10^{-3} \mathrm{kgmel}$ \\
\hline B & $c_{3}$ coefficient & 5.82 calimol \\
\hline $\mathrm{C}$ & $c_{p}$ coefficient & $0.78 \mathrm{cal} / \mathrm{mol}$ \\
\hline$b$ & Burgers vector & $2.86 \times 10^{-10} \mathrm{~m}$ \\
\hline$k / b^{3}$ & Boitemann's constant divided by $\hat{z}^{2}$ & $59 \times 10-\mathrm{GPaK}$ \\
\hline$p$ & Obstacle activation energy exponen: & 33 \\
\hline 9 & Obstacle activation energy exponent & 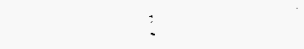 \\
\hline$\dot{\varepsilon}_{0}$ & Strain-rate pre-exponentia! & $10, \mathrm{~s}^{-i}$ \\
\hline 300 & Dimensionless obstacie activation: $8 \alpha 0=G_{00} / 4(295 \mathrm{~K}) \mathrm{c}^{3}$ & $0.59+$ \\
\hline
\end{tabular}
Fig. 1. Parameter values are collected in Tables 1 and 2, and the curve for the function $f$ in Eq. (3) is shown in Fig. 2. We take $P=1$ in Eq. (2).

TABLE 1. Physical Constants and Non-adiustable Model Parameters 
TABLE 2. Adiustable Model Parameters

\begin{tabular}{cccl}
\hline Parameter & Units & Equation/Definition & Value \\
\hline$\sigma_{\mathrm{a}}$ & $\mathrm{GPa}$ & $\sigma_{\mathrm{i}}=\sigma-\sigma_{\mathrm{a}}$ & 0.055 \\
$\mathrm{~g}_{\mathrm{p} 1}$ & - & $g_{\mathrm{p} 1}=G_{\mathrm{p} 1} / \mu(T) b^{3}$ & 0.0847 \\
$\hat{\sigma}_{\mathrm{p} 1}$ & $\mathrm{GPa}$ & 3 & 1.4 \\
$g_{\mathrm{p} 2}$ & - & $g_{\mathrm{p} 2}=G_{\mathrm{p} 2} / \mu(T) \hat{\sigma}^{3}$ & 0.0344 \\
$\hat{\sigma}_{\mathrm{p} 2}$ & $\mathrm{GPa}$ & 3 & 0.056 \\
$\hat{\theta}_{\mathrm{p} 2}$ & $\mathrm{GPa}$ & 7 & 6.2 \\
$\hat{\sigma}_{\mathrm{p} 10}$ & $\mathrm{GPa}$ & 7 & 1.3 \\
$\hat{\theta}_{\sigma}$ & $\mathrm{GPa}$ & 5 & 6.8 \\
$\hat{\sigma}_{\mathrm{os} 0}$ & $\mathrm{GPa}$ & 6 & 0.605 \\
$\mathrm{~A}$ & - & - & 0.118 \\
\hline
\end{tabular}

\section{RESULTS AND COMPARISON TO EXPERIMENTAL DATA}

Ingots of unalloyed tantalum* were forged, rolled, and annealed to yield material with a uniform grain size of 35 to $38 \mu \mathrm{m}$. Cylindrical specimens $0.5 \mathrm{~cm}$ in dia. by $0.5 \mathrm{~cm}$ high were prepared and tested on a conventional hydraulic test machine and a compressive split Hopkinson pressure bar (SHPB).

Calculations at $298 \mathrm{~K}$ with constant $\hat{\sigma}_{\mathrm{p} !}$ and $\hat{\sigma}_{\mathrm{p} 2}$ are compared with our data in Fig. 3 . The model reproduces the initial yield stresses very well, and at large strains, the agreement remains reasonably good because obstacle hardening eventually becomes the dominant mechanism. At smaller strains where the Peieris mechanism dominates, however, plateaus of constant flow stress much wider that those observed dominate the calculated flow curves. To remedy this, we allowed the threshold stress $\hat{\sigma}_{p 2}$ in Eq. (3) to increase with deformation according to

$$
\frac{d \hat{\sigma}_{p 2}}{d \varepsilon}=\theta_{p 2}\left(1-\frac{\hat{\sigma}_{p 2}}{\hat{\sigma}_{p 20}}\right)^{0.70} .
$$

This expression has no physical meaning of which we are aware, and we have selected it because it has a form consistent with data at $77 \mathrm{~K}$ (Fig. 4).

Flow curves recalculated with this modification are compared with the experimental data in Figs. 4 and 5. At room temperature (Fig. 5), the improve. ment in the agreement at intermediate strains is striking. Similarly, the description of the SHPB results (Fig. 4) is much improved, with modes: hardening controlled by Eq. (7) a prominent feature.

\footnotetext{
* Cabot Corporation, Boyerstown, Pennsylvania. LSA
}

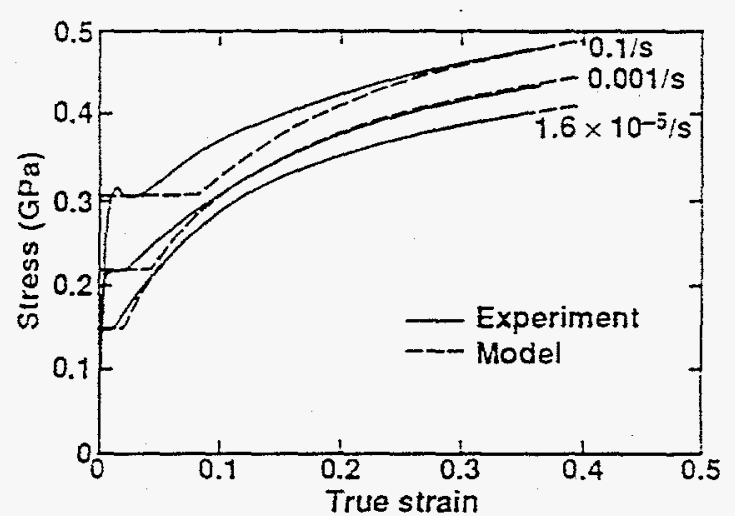

FIGURE 3. Comparison of model calculations for constant $\hat{\sigma}_{\text {, }}$ and $\hat{\sigma}_{\beta}$ with experimental observations for various strain rates at $298 \mathrm{~K}$. Calculations assume deformation take place isothermally.

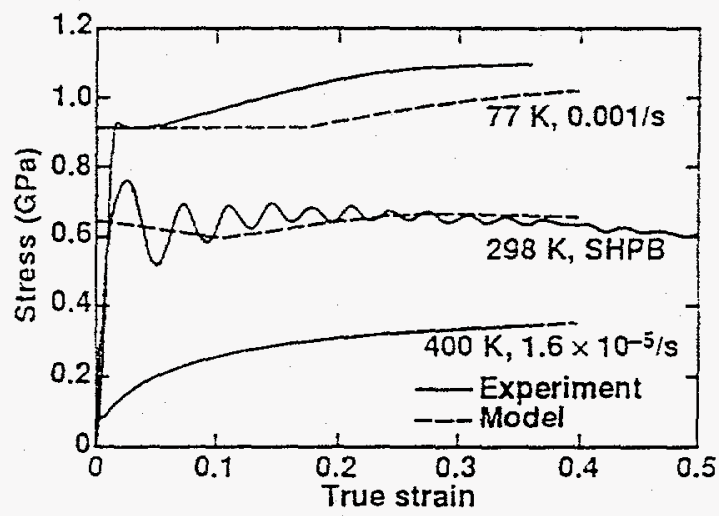

FIGURE 4. Comparison of model calculations for constant $\hat{\sigma}_{71}$ and variable $\hat{\sigma}_{\text {, [Eq. (7)] with experimenta] observations for }}$ high and low strain rates at various temperatures. Deformations at 400 and $77 \mathrm{~K}$ are assumed to take place isothermally, but the SHPB experiment is assumed to be adiabatic.

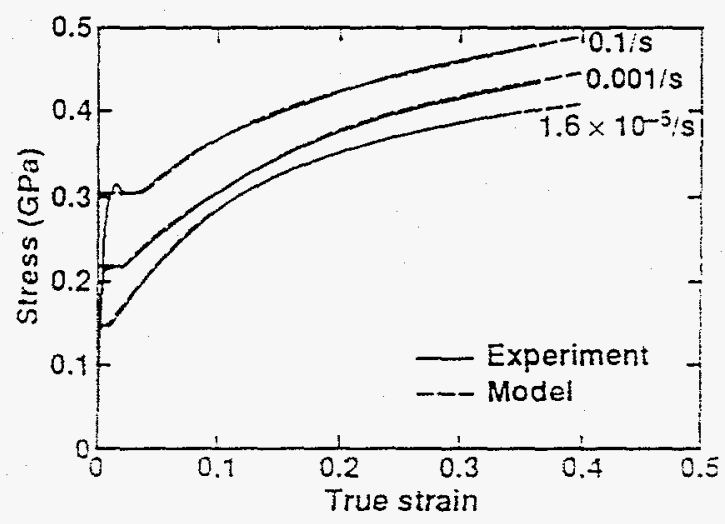

FIGLRE 5. Comparison of model calculations for constant $\dot{\sigma}_{2}$ and variabie $\hat{\sigma}_{22}[$ [Eq. (f)] with experimental observations for various strain rates at various temperatures. Calcuiations assume deformation takes piace isothernally. 
Some hardening of the flow stress at $77 \mathrm{~K}$ is also apparent, but here the agreement is not completely satisfactory. As expected, the introduction of Eq. (7) has no effect on the fiow curve at $400 \mathrm{~K}$ (Fig. 4) because the obstacle term dominates all but a very limited initial flow regime.

\section{DISCUSSION}

Our model describes the initial flow stress of tantalum well over a wide range of strain rate and a modest range of temperature. Although the curve for $G_{p}$ given in Fig. 2 falls slightly above the data of Conrad (1), the agreement is acceptable, in view of the sensitivity of the flow stress of tantalum to solutes and interstitials. Certainly the value of $G_{p 1}$ that we find (Table 2) is within the range of values reported in the literature $(2,8-10)$.

Screw disiocations are a characteristic of the microstructure of deformed bec metals and are known to carry much of the plastic deformation, particularly at low temperatures $(10,11)$. While this provides a structural rationalization for the dominance of the Peieris mechanism in determining the initial flow stress in tantalum, the secondary term proposed in Eq. (3) has no such obvious analog. One plausible suggestion is the noncoplanar dissociation of the dislocation core $(2,8)$, but the magnitude of the activation energy associated with such a mechanism has not been estimated. Furthermore, it is not clear why $\sigma_{p 2}$ should increase with increasing deformation. Nevertheless, calculations clearly show that our model can describe at least semi-quantitatively the crossing of the hardening curves for unalloyed tantalum noted in (4). It thus may provide a naturai explanation for this otherwise puzzling behavior of the hardening as a function of strain rate.

At low strain rates or elevated temperatures, obstacles appear to dominate the flow curves after initial yield. The shape of such flow curves, determined by Eq. (5), applies equally well to both bec and fcc metals. This suggests that the mechanism of plastic flow, broadly speaking, is the same for both materials under these deformation conditions.

\section{CONCLUSIONS}

Our model provides a plausible framework tor describing the well-known yield behavior of bec metals and their hardening in an integrated and physically motivated manner. From this work, we conclude the following:
- Deformation of unalloyed tantalum is controlled by a series combination of (1) a "Peierls" mecinanism with a small activation energy $(0.5-1.0 \mathrm{eV})$ and a large threshold stress ( $-1.0 \mathrm{GPa}$ ) and (2) an "obstacle" mechanism with a large activation energy $(\sim 10 \mathrm{eV})$ and a small threshold stress that increases with deformation.

- The Peierls term consists of a larger and dominant component $(0.85 \mathrm{eV})$ associated with a constant threshold stress (1.4 GPa) and a smaller component $(0.35 \mathrm{eV})$ associated with an initially small threshold stress.

- To reproduce the flow behavior of unalloyed tantalum at intermediate strains, this secondary threshold stress must increase with deformation.

- At very low strain rates or elevated temperatures, hardening of unalloyed tantalum can be described by the same relationships as obstacle hardening in fcc metals.

\section{ACKNOWLEDGMENTS}

We thank M. LeBlanc and D. Shields for performing the experiments for this study. Work was performed under the auspices of the U.S. Department of Energy by the Lawrence Livermore National Laboratory under Contract No. W-7405-Eng-48.

\section{REFERENCES}

1. Conrad, H., "The cryogenic properies of metals," in HighStrength Materials, V. F.Zackay, ed. New York, Wiley, 1965), Ch 11, pp. 436-509.

2. Hoge, K. G., and Mukherjee, A. K., J. Moter. Sci. 12. 1666-1672 (1977).

3. Christian, J. W. Metall. Trans. A 14A 1237-1256 (1983)

4. Gourdin, W. H., Lassiła, D. H., LeBlanc, M. YI., and Shields, A. L., J. Phys. (Paris) IV CS, 207-212 (1994).

5 Chen, S. R., and Gray, G. T., III, "Constituive behavior of tungsten and tantalum, experiments and modeling," Los Alamos Nacional Latoratory. Los Alamos, NM, LA-CR94-3405, presented at 1994 International Conference on Tungsten and Refractory Mctals, McLean VA. Octocer i:$19,1994$.

5. Follansbee, P. S., and Kocks, L. F.. Acta Metcil. 36, \$1-9:3 (1988).

7. Gourdin, W. H., and Lassila, D. H., Acta Letcil. et Water. $39,2337-2348$ (1991).

3. Christian, J. W., and Masters, B. C., Proc. Rov. Soc. A 281 240-257 (1964).

․ Wemer, .M., Phys. Stat. Sol. 104, 63-78 (1987).

10. Mizubayashi, H., Egashira, H., and Okuda, S. Acta Meto:: et Mater. 43 (1) 269-276 (1994).

$\therefore$ Johnson, P. E., "The Stnctural Dependence of Work Hardening in Low Carbon Steels," Luwrence Berkeiey Laboratory, Berkeley, CA. LBL-31670 199: 